\title{
Design of a Wideband Radio Telescope
}

\author{
William A. Imbriale \\ Jet Propulsion Laboratory, 4800 Oak Grove Drive, Pasadena, CA 91109 \\ William.A.Imbriale@jpl.nasa.gov / 818-354-5172 \\ Sander Weinreb and Handi Mani \\ California Institute of Technology, 1200 East California Blvd., Pasadena, CA 91125 \\ Sander.Weinreb@jpl.nasa.gov / 818-354-4065
}

\begin{abstract}
A wideband Radio Telescope is being designed for use in the Goldstone Apple Valley Radio Telescope program. It uses an existing 34-meter antenna retrofitted with a tertiary offset mirror placed at the apex of the main reflector. It can be rotated to use two feeds that cover the 1.2 to $14 \mathrm{GHz}$ band. The feed for 4.0 to $14.0 \mathrm{GHz}$ is a cryogenically cooled commercially available open boundary quadridge horn from ETS-Lindgren. Coverage from 1.2 to 4.0 $\mathrm{GHz}$ is provided by an un-cooled scaled version of the same feed. The performance is greater than $40 \%$ over most of the band and greater than $55 \%$ from 6 to $13.5 \mathrm{GHz} .{ }^{12}$
\end{abstract}

\section{TABLE OF ConTEnTS}

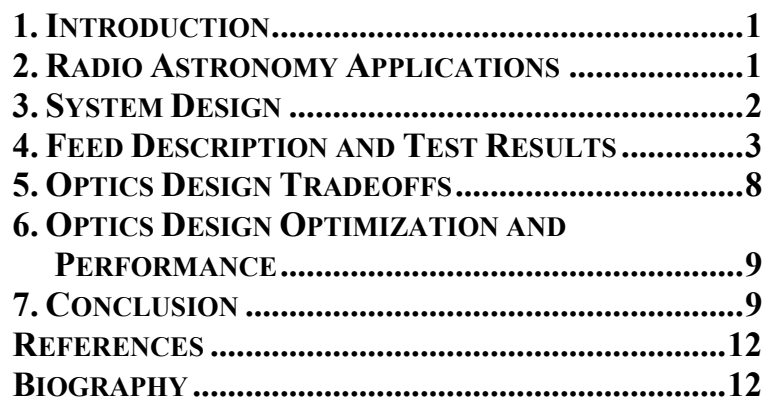

\section{INTRODUCTION}

The Goldstone Apple Valley Radio Telescope (GAVRT) outreach project is a partnership involving NASA, the Jet Propulsion Laboratory (JPL), the Lewis Center for Educational Research (LCER), and the Apple Valley Unified School District., located east of Los Angles near the NASA Goldstone deep space communication complex. This educational program currently uses a 34-meter antenna, DSS12, at Goldstone for classroom radio astronomy observations via the Internet. The GAVRT program [1] introduces students in elementary, middle, and high school to the process of science with the goal of improving science literacy among American students. The current program utilizes DSS12 in two narrow frequency bands around Sband $(2.3 \mathrm{GHz})$ and $\mathrm{X}$-band $(8.45 \mathrm{GHz})$ and is heavily subscribed by a training program involving a large number of secondary school teachers and their classrooms. To expand the program, a joint JPL/LCER project was started in mid $2006 \mathrm{t}$ to retrofit an additional existing 34-meter beam-waveguide antenna, DSS28, with a wide band feed and receiver to cover the 1.2 to $14 \mathrm{GHz}$ frequency bands.

\footnotetext{
${ }^{1} 1-4244-0525-4 / 07 / \$ 20.00$ @2007 IEEE.

2 IEEEAC paper\#1586, Version 3, Updated 2007:01:30
}

The antenna to be retrofitted was designed as part of the JPL Antenna Research System Task described in reference [2]. The antenna, shown in Figure 1, has a 34-meter diameter main reflector, a 2.54 meter subreflector and a set of beam waveguide mirrors surrounded by a 2.43 meter tube. The antenna was designed for high power and a narrow frequency band around 7.2 GHz. The performance at the low end of the frequency band desired for the educational program would be extremely poor if the beam waveguide system was used as part of the feed system. Consequently, the revised design uses a wideband feed illuminating a tertiary mirror positioned near the apex of the main reflector. This paper will describe the wideband radiometric receiver front end with emphasis on the feed and optical system.

\section{RADIO ASTRONOMY APPLICATIONS}

A key principle of the GAVRT outreach program is the involvement of JPL and other scientists in terms of interest in the data and contact with the teachers through the Lewis Center. This involvement is greatly enhanced by having professional quality instrumentation that is capable of providing new results of significant scientific value. For this reason the expansion program will equip DSS28 with radiometers which have state-of-the-art sensitivity over an unprecedented decade of more of frequency range. The observations that are enabled by the 1.2 to $14 \mathrm{GHz}$ frequency range are:

(1) Expansion of the existing DSS12 S and X band observation of the time variation of radio radiation from Jupiter.

(2) Observations of the broadband continuum radio emission from other planets, quasars, supernova remnants, and other astronomical objects where the intensity as a function of frequency gives important information about the physics of the radiation.

(3) Observations of the $1.42 \mathrm{GHz}$ hydrogen line [3]. Hydrogen is the most abundant element in the universe, can be observed in most interstellar regions of our galaxy, and by measurements of the Dopplershifted line can be used as a probe of radial velocity and radio luminosity of other galaxies. A receiving system capable of $1.2 \mathrm{GHz}$ observations will allow measurements of galaxies with red-shifts up to 0.15 of the velocity of light. 

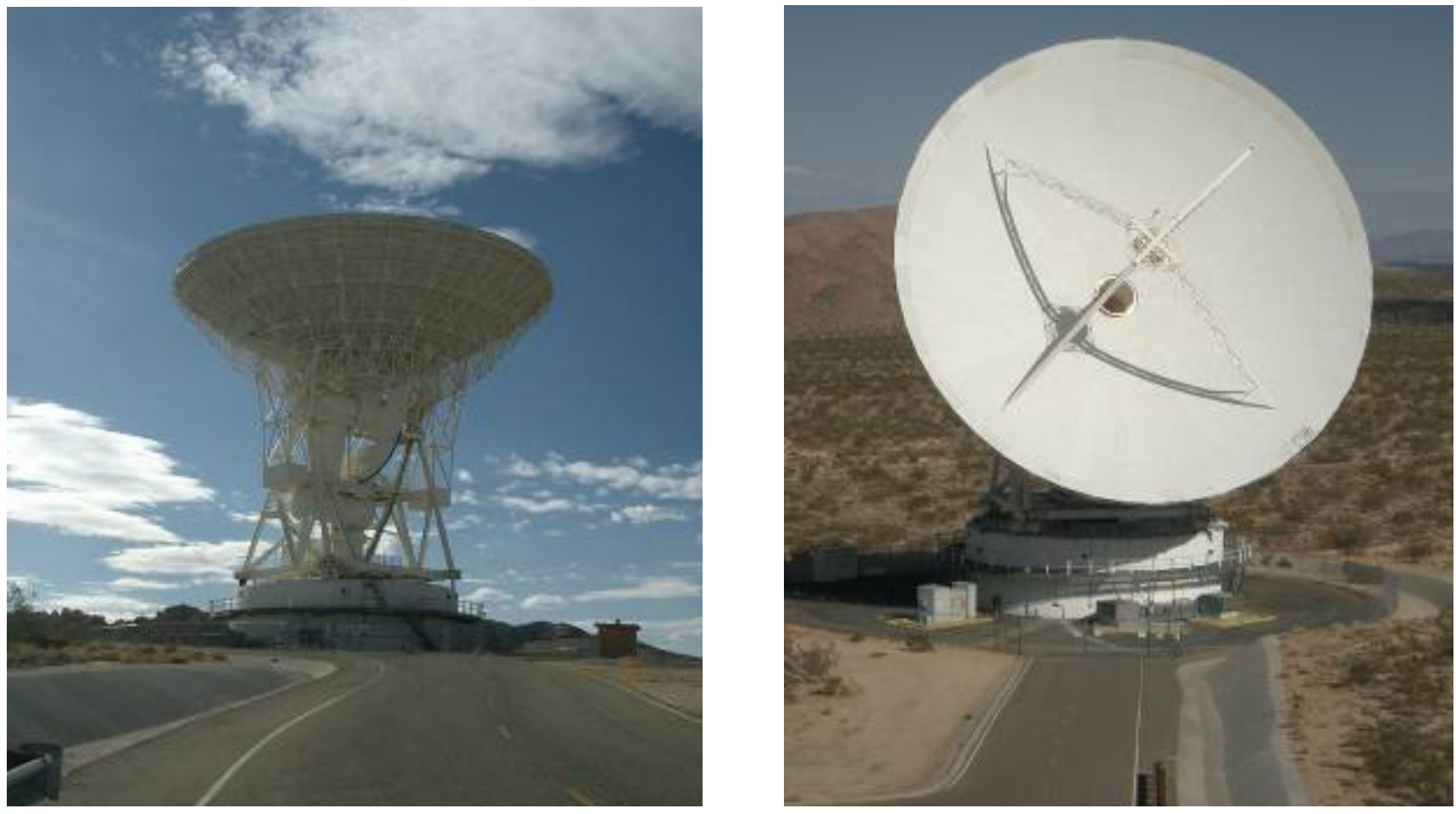

Figure 1 - Photographs of the DSS27 antenna which is identical to the DSS28 which will be modified for the wide band operation described in this paper. The original antenna has a beam-waveguide optical system which guides the beam through the $2.74 \mathrm{~m}$ diameter hole at the vertex, through mirrors, to a large stationary control room in the antenna base. The revised system will utilize a tertiary reflector covering hole with receivers located near the vertex.

(4) Observations of many radio astronomy molecular lines such as hydroxyl $(1.67 \mathrm{GHz})$, methyladyne $(3.3 \mathrm{GHz})$, formaldehyde (4.8 and $14.5 \mathrm{GHz})$, methanol (6.7 and $12.2 \mathrm{GHz})$, helium $(8.7 \mathrm{GHz})$, acetamide $(9.2 \mathrm{GHz})$, and cyclopropenone $(9.3 \mathrm{GHz})$. The strengths and line shapes of these lines allow study of the chemistry in distant region of the universe.

(5) Observations of pulsars, in particular, pulses of subnanosecond duration recently discovered by Hankins [4] and requiring wide bandwidth to measure the pulse width. The timing and intensity of many other pulsars are of great interest as probes of gravitational waves and physics of neutron stars.

(6) Search for signals from extra-terrestrial civilizations. It is to be expected that public and scientific interest in this topic will be greatly enhanced by the discovery of earth-like planets by the Kepler spacecraft [5] by 2011 .

\section{SYSTEM DESIGN}

The complete system involves the reflector, subreflector, tertiary reflector, feed, cryogenics subsystem, low noise amplifiers, noise calibration system, frequency converters, digital spectrometers, continuum signal processing, and monitor and control system. Only the tertiary, feed, cryogenics, and LNA will be discussed in this paper. The main parameters of this front-end are the antenna efficiency, $\eta$ and the system noise temperature, Tsys. Our goal is an $\eta$ of $>40 \%$ from 1.2 to $14 \mathrm{GHz}$ with typical values of $55 \%$, and a maximum Tsys of $55 \mathrm{~K}$ over the band with $35 \mathrm{~K}$ at best frequencies. These values of Tsys include $2.7 \mathrm{~K}$ cosmic background, $.3 \mathrm{~K}$ of atmosphere, and $.5 \mathrm{~K}$ allocated for spillover and feed support reflections of ground radiation so $10 \mathrm{~K}$ must be added the noise temperature of feed and LNA.

In order to meet the above Tsys requirement we believe the wideband feed (which has more loss than narrow band feeds) needs to be cryogenically cooled, at least for frequencies above $3 \mathrm{GHz}$. A single compact wideband feed covering the 1.2 to $14 \mathrm{GHz}$ is under development by Kildal at Chalmers University (see [6] ) and may be utilized but, in the next section, we describe a baseline approach with a commercially available feed to cover the 4 to $14 \mathrm{GHz}$ range. In this case, a second feed, which may not be cryogenically cooled, could be a scaled in size by 3.5 to cover 1.14 to $4 \mathrm{GHz}$.

The wideband LNA's required for the system have been under development for many years and over 100 units of the type shown in Figure 2 have been assembled, tested at 15K, and utilized in radio astronomy and physics research systems. When cooled to $15 \mathrm{k}$, the noise is under $5 \mathrm{~K}$ from 1 to $12 \mathrm{GHz}$ when driven from a $50 \mathrm{ohm}$ generator. The computer noise model is available and can be used to determine and optimize the noise for other impedances presented by the feed. 

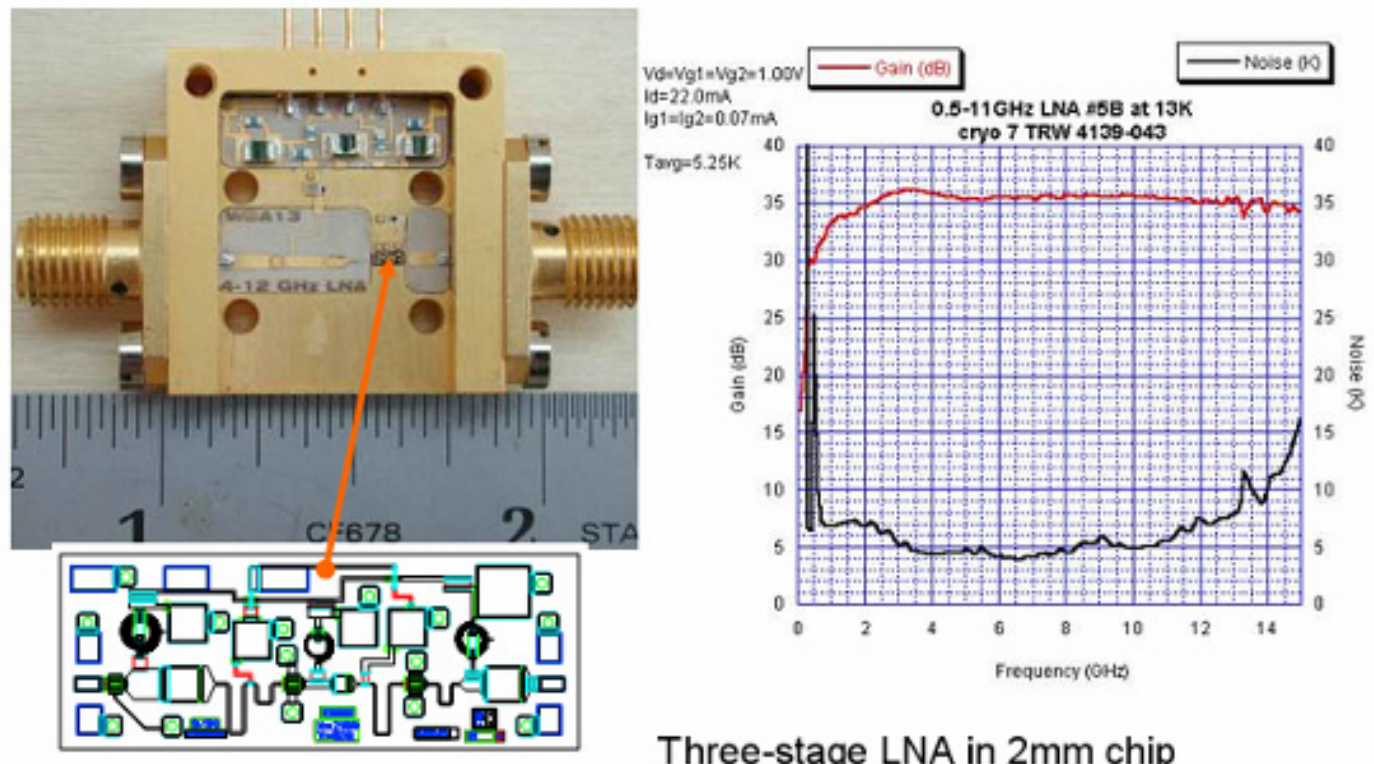

Three-stage LNA in $2 \mathrm{~mm}$ chip

Figure 2 - Cryogenic low noise amplifier used in the wideband system. The amplifier was designed at Caltech and the 0.1um InP HEMT MMIC is fabricated by Northrop Grumman. The noise is under $7 \mathrm{~K}$ and gain over $35 \mathrm{~dB}$ form 1 to $12 \mathrm{GHz}$ when cooled to $15 \mathrm{~K}$. The input $1 \mathrm{~dB}$ gain compression point is above $-40 \mathrm{dBm}$ and the power consumption is $22 \mathrm{~mW}$. Over 100 of these LNA's have been constructed and are in use.

\section{FEED DESCRIPTION AND TEST RESULTS}

Pattern and noise measurements of the ETS-Lindgren Model 3105-64 antenna designed by V. Rodriguez [7], will be described in this section. Integration of the feed with the cryogenics dewar can have a large effect upon the performance and the mechanical configuration is shown in Figure 3. Other than Teflon in the SMA connector the feed is constructed entirely of aluminum and no deleterious affects of the cryogenic cooling are expected. (The red polycarbonate rims seen in Figure 3 lower-left serve no purpose and were removed for all tests.) Within the outer dewar aluminum cylinder vacuum jacket there is an aluminum radiation shield of diameter $24.1 \mathrm{~cm}$ to prevent thermal coupling of the feed to $300 \mathrm{~K}$. Thermal radiation, of the order of $20 \mathrm{~W}$, enters the window but is mostly blocked by a blanket consisting of 16 layers of $25 \mathrm{um}$ thick Teflon film separated by a mesh of fine French wedding veil. Without this blanket it was not possible to cool the feed below $100 \mathrm{~K}$ but with the blanket the temperature measured on the feed was $21 \mathrm{~K}$. The total thickness of Teflon is thus $0.4 \mathrm{~mm}$ and with dielectric constant of 2.1 the total electrical length is $1 / 38$ at $14 \mathrm{GHz}$ which will produce negligible reflections. The window material is $1.5 \mathrm{~mm}$ thick highdensity polyethylene (HDPE) with dielectric constant of 2.4. The window electrical length of $1 / 9.2$ at $14 \mathrm{GHz}$ is not negligible but will not have a large effect upon the measured noise. The total force on the window at $15 \mathrm{psi}$ atmospheric pressure is $1140 \mathrm{lbs}$ which produces a perimeter radial stress of 588 psi compared to a 4270 psi yield stress for HDPE. Thus, a better compromise may be a thinner window but cold flow and creep will also need to be considered.
The feed return loss without window was measured as shown in Figure 4. The $2.4 \mathrm{GHz}$ ripple period in the data would arise from two reflections spaced $6.2 \mathrm{~cm}$ apart which is of the order of the spacing of the connector to the radiating region of the slot. The feed has a built-in balun and could be better matched with a differential LNA. Effects of the feed impedance variation are observed in the noise data and improvements in the noise match to the LNA for particularly important frequencies could be implemented.

The feed patterns were measured with four different configurations of surrounding structures as shown in Figure 5 with results compared in Figure 6. It was found that the pattern for illumination of a reflector could be improved by surrounding the feed with an absorbing cylinder. At a later stage it was realized that placing absorbing strips of lossy material on the outer surfaces of the fins could have the same effect. Currents flowing around the outer perimeter of the fins are thus absorbed and prevented from coupling to the surrounding cylinder. The question is raised as to whether the absorber will deteriorate the efficiency of the feed and add to the noise temperature. Data indicating that the efficiency may not be reduced is shown in Figure 7 where the transmission loss from the feed to a test antenna in an anechoic chamber is measured. The result shows a decrease in on-axis loss at frequencies below $3 \mathrm{GHz}$, small increase in loss from 3 to $6 \mathrm{GHz}$, and negligible effects above $6 \mathrm{GHz}$. The noise temperature contributed by the absorber should be small if the absorber is at cryogenic temperatures. 

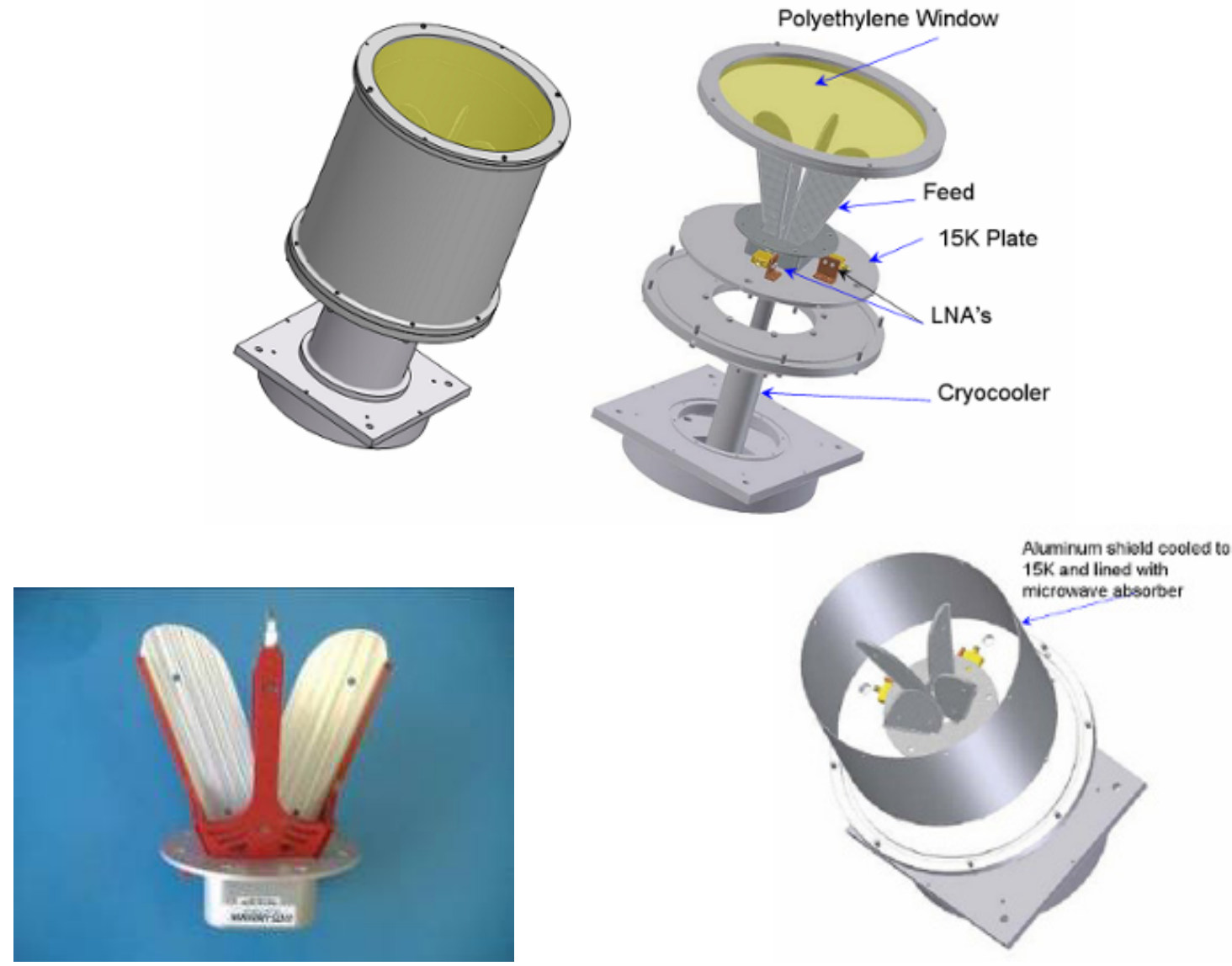

Figure 3 - Integration of the ETS-Lindgren feed in a cryogenic dewar with $30 \mathrm{~cm}$ outside diameter. The cut-a-way view at upper right shows the components with the $15 \mathrm{~K}$ radiation shield and vacuum dewar cylinder removed. The $15 \mathrm{~K}$ shield is shown at lower right.

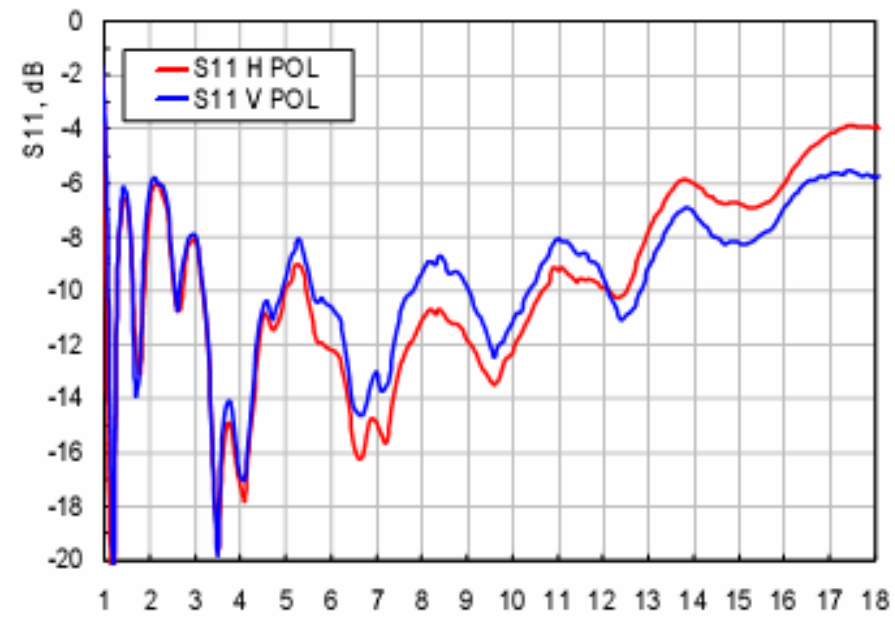

Figure 4 - Return loss of the two linear ports of the feed. The pattern data is of the $\mathrm{H}$ port. 
Feed in aluminum $25 \mathrm{~cm}$ diameter cylinder to simulate cryogenics dewar
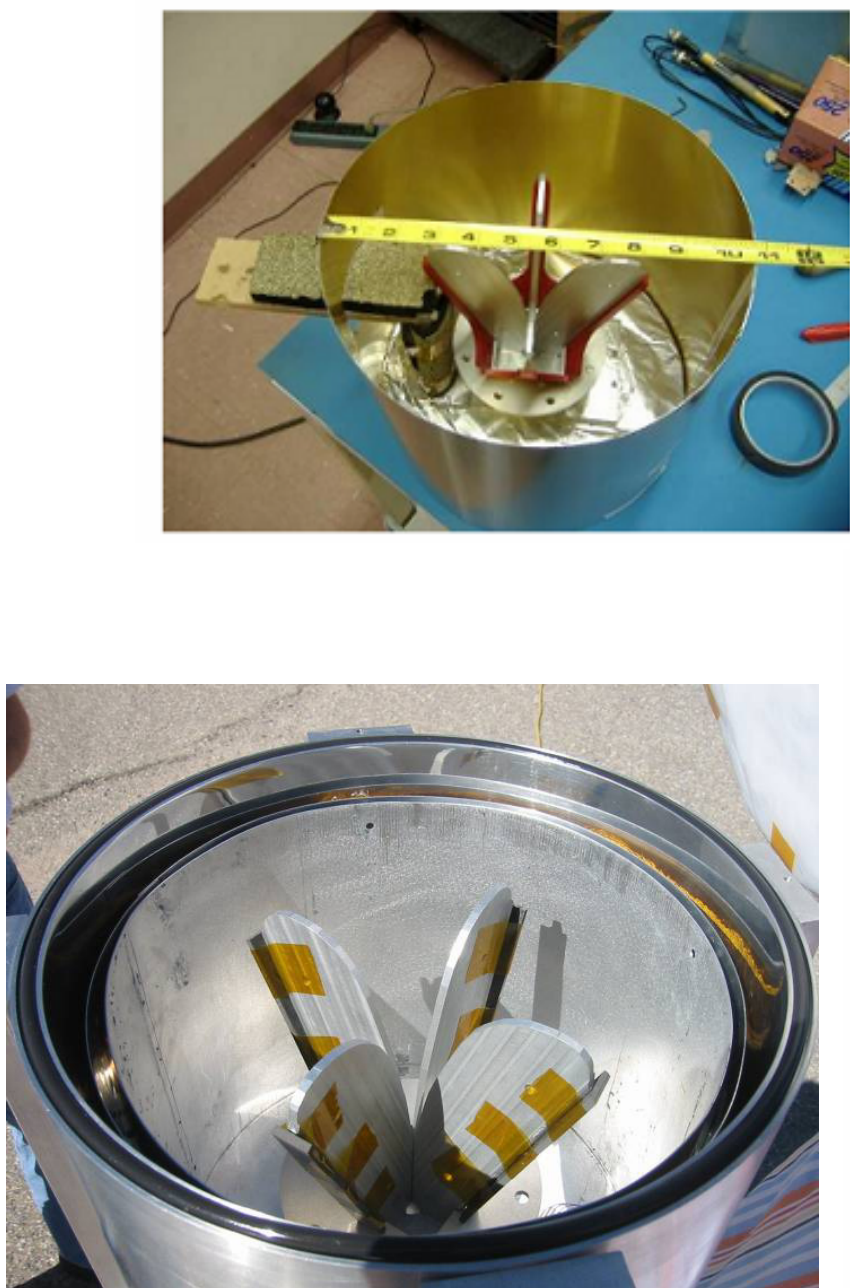

Absorber material inserted in cylinder for additional tests

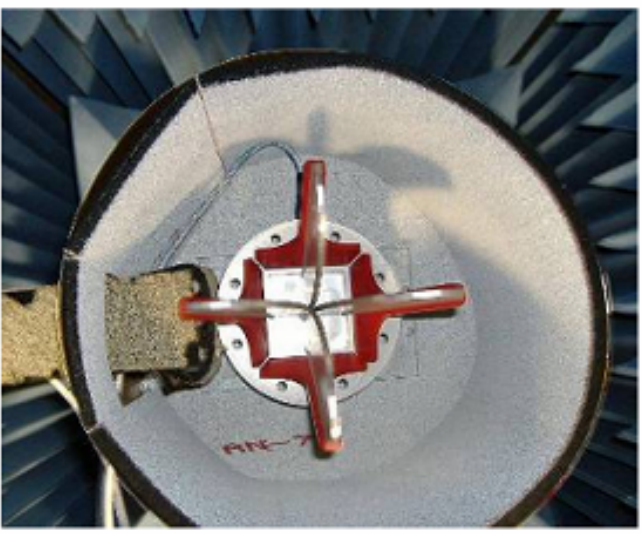

Test Transmitting Antenna Approximately $1 \mathrm{~m}$ From Feed Under Test - Rotated $90^{\circ}$ for Photograph

Figure 5 - Feed in dewar and in pattern test chamber. The feed patterns were tested with a surrounding cylinder at upper left, the cylinder line with absorber at upper right, and in the cylinder but with absorbing strips taped to the outer rim as in lower left. All pattern measurements were made in a small chamber at Caltech as shown in lower right, where is this photo, the feed without cylinder or absorber was tested. The efficiency calculations were performed from pattern measurements with the feed with absorber strips and in a cylinder as in lower left 

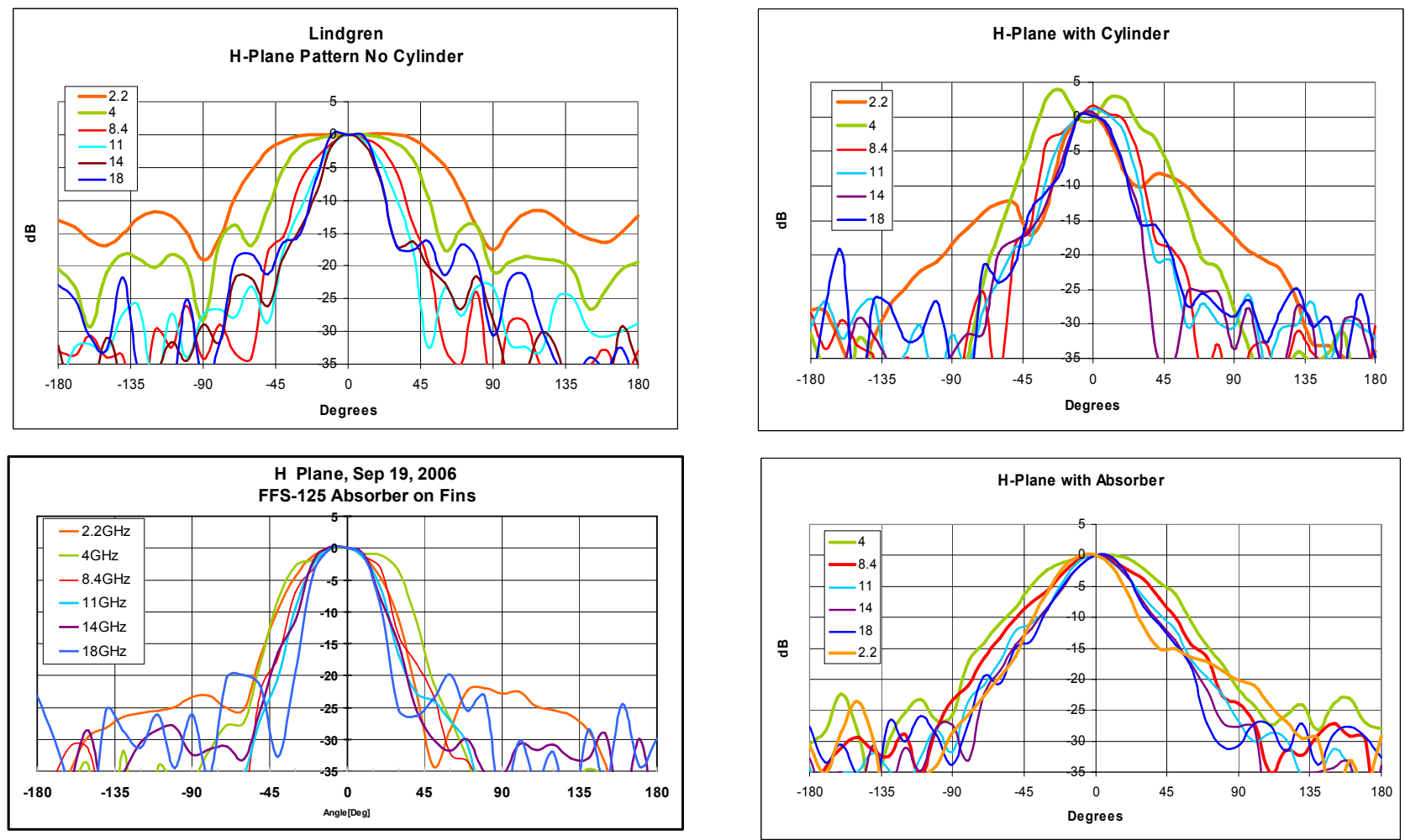

Figure 6-H plane patterns of the feed at 6 frequencies and 4 types of enclosures: Top left, feed alone, Top right, feed in cylinder, lower right, feed in cylinder with absorber on walls, and lower left, feed in cylinder with absorber strips on outside edge of fins. Note the narrowing of the pattern and reduction of frequency variation as the absorber is added.

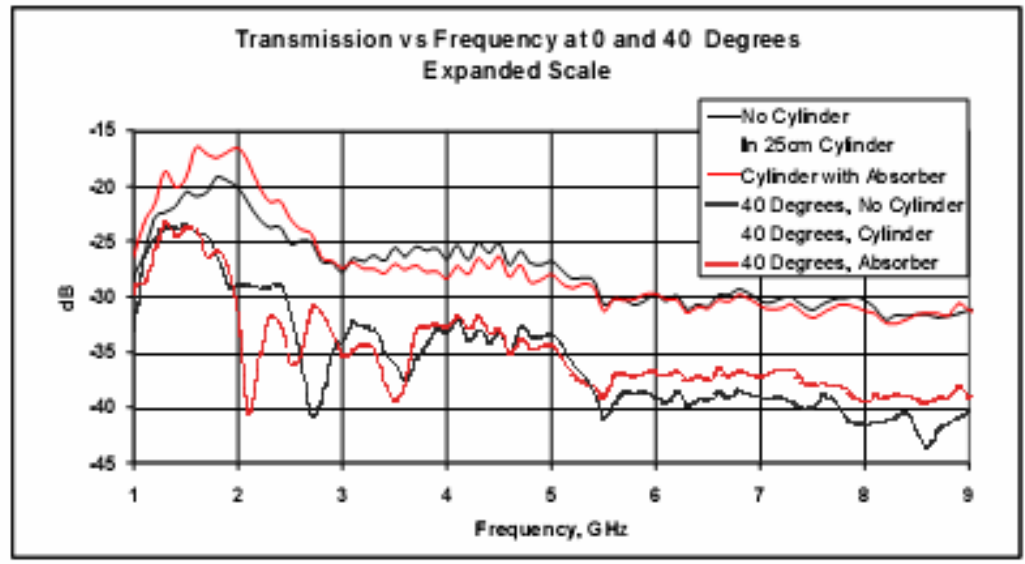

Figure 7 - Transmission in anechoic chamber from transmitting antenna to ETS-Lindgren feed vs frequency with and without the surrounding cylinder lined with absorber. Curves both on-axis and 40 degrees off axis are shown. Note that below $3 \mathrm{GHz}$ the on-axis transmission is enhanced by approximately $3 \mathrm{~dB}$ when the absorber is added.

After the pattern tests the feed was integrated with LNA's on both polarizations and a cryogenics dewar cooled with a closed-cycle CTI Model 350 cryocooler. This cooler has a capacity of $2 \mathrm{~W}$ at $14 \mathrm{~K}$ and $5.5 \mathrm{~W}$ at the measured temperature of $21 \mathrm{~K}$. Cool-down time was approximately 6 hours.

Noise temperature tests of the cooled system were performed both in Pasadena, $\mathrm{CA}$ and later in the Mohave desert at Goldstone, CA, Figure 8, to reduce the effects of radio frequency interference. (RFI). One polarization had a 4-12 GHz LNA, \#87D, with $39 \mathrm{~dB}$ gain and $<10 \mathrm{~K}$ noise from 3 to $14 \mathrm{GHz}$ increasing to $20 \mathrm{~K}$ noise at $2 \mathrm{GHz}$ while the other polarization had a modified $0.5-11 \mathrm{GHz}$ LNA (labeled the 2-12 GHz LNA), \#80, also with $39 \mathrm{~dB}$ gain and $<10 \mathrm{~K}$ noise from 3 to $14 \mathrm{GHz}$ but with $15 \mathrm{~K}$ noise at $2 \mathrm{GHz}$. As a check of overloading due to RFI the total power out of the LNA's was measured with values in the $-40 \mathrm{dBm}$ range at Goldstone and $-20 \mathrm{dBm}$ in Pasadena. With $39 \mathrm{~dB}$ of LNA 


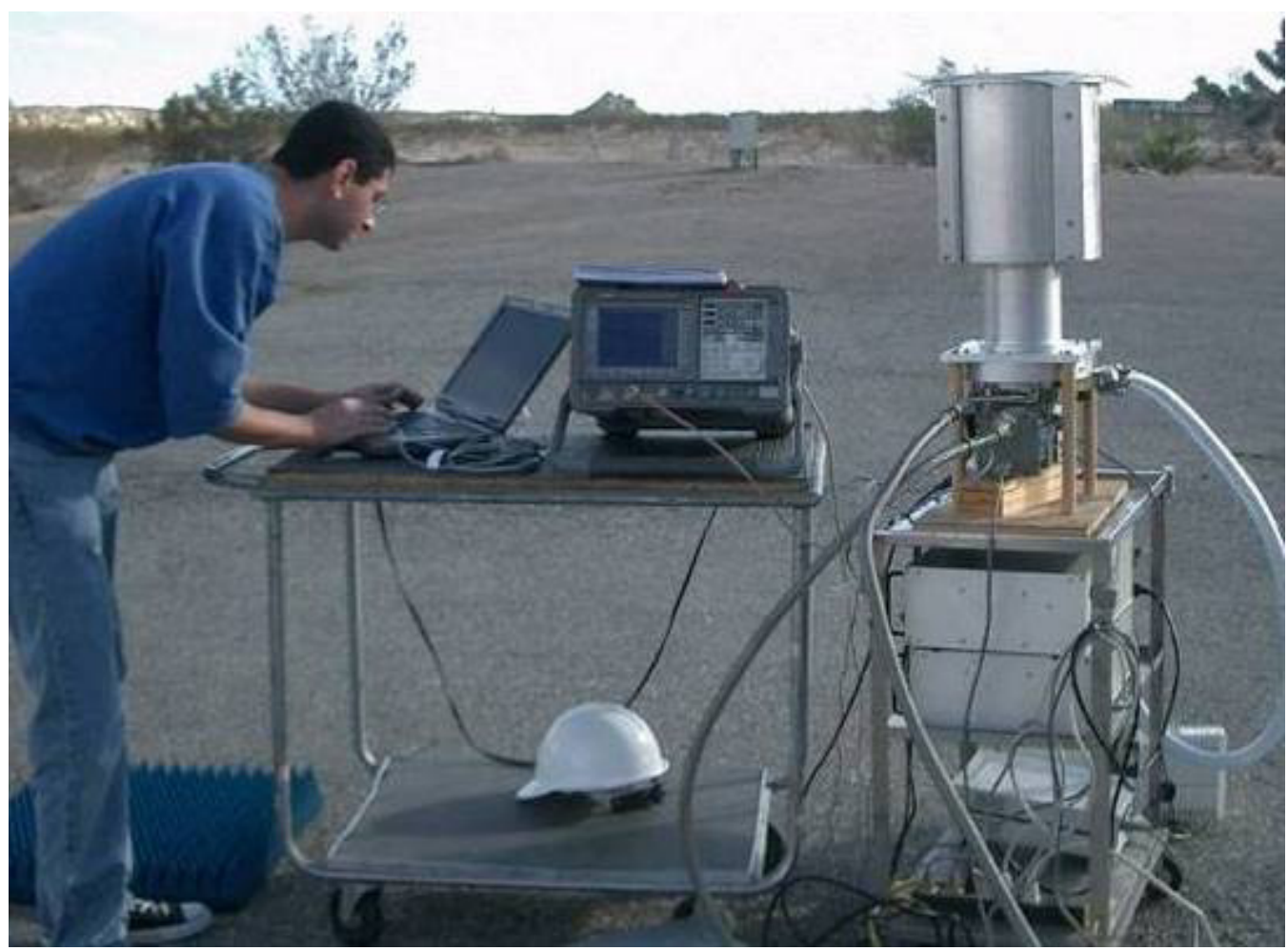

Figure 8 - Test setup for noise measurements of the cooled feed at Goldstone on Oct 10, 2006 using cold sky and absorber (lower left) on top of the feed window.

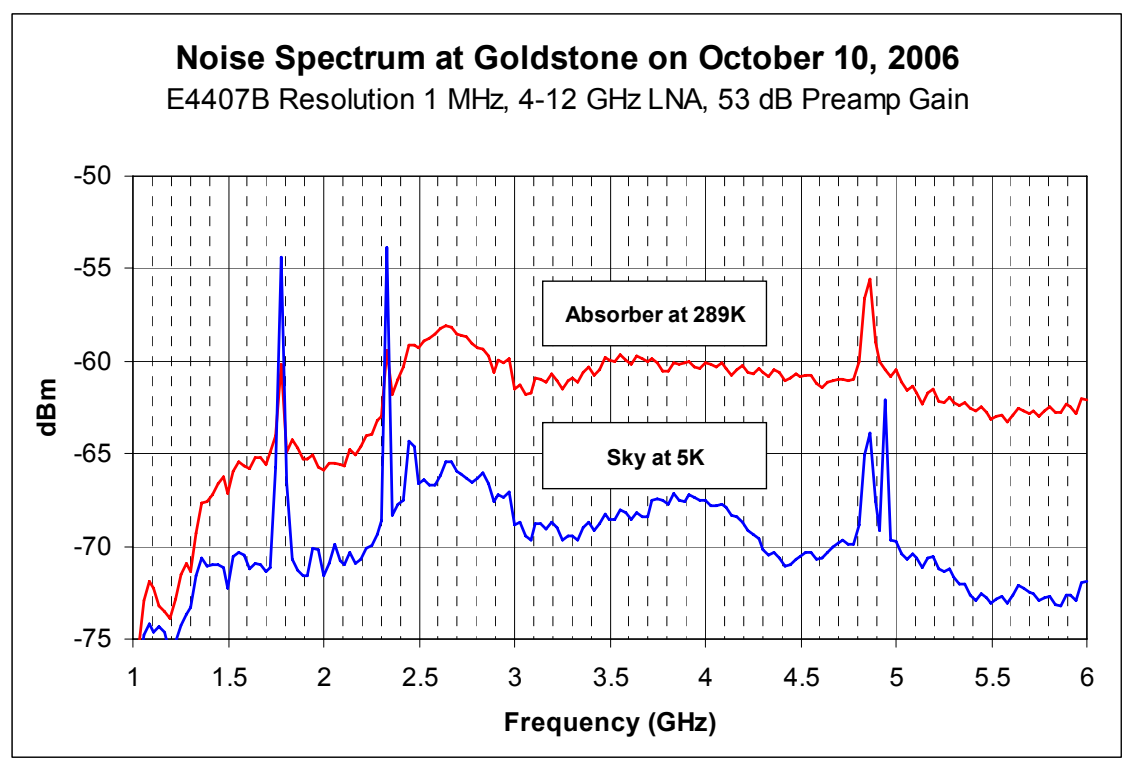

Figure 9 - Raw data spectrum analyzer plots of LNA output power with absorber covering the feed and with the feed viewing cold sky. A ratio, Y-factor, of $9 \mathrm{~dB}$, gives a noise temperature of $36 \mathrm{~K}$. The spikes are due to RFI. 


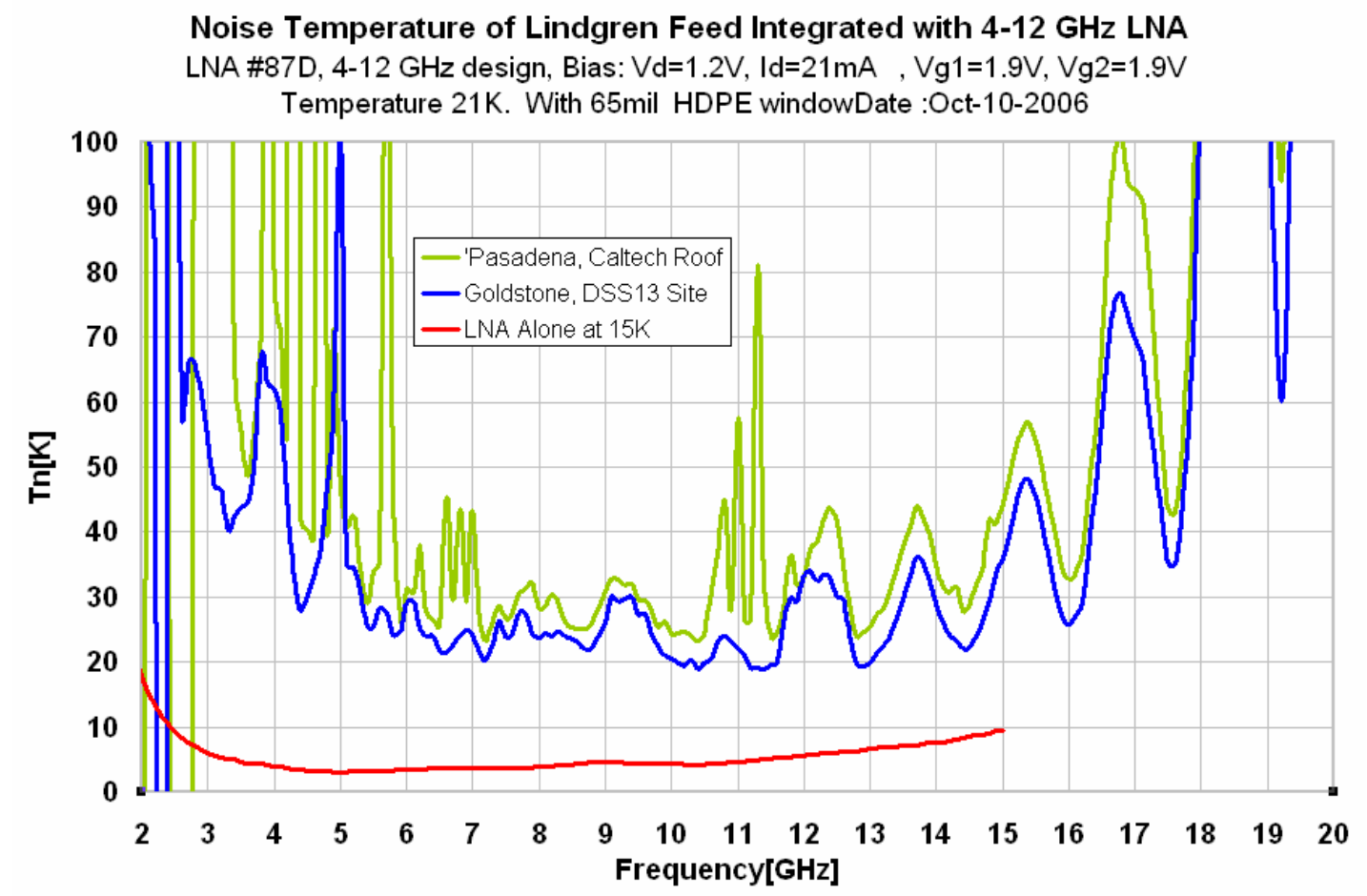

Figure 10 - Noise temperature of feed and LNA as a function of frequency for measurements in Pasadena (top), Goldstone, and of the LNA alone measured at $15 \mathrm{~K}$ referred to its coaxial input jack. The noise is under $35 \mathrm{~K}$ from 4.3 to $15 \mathrm{GHz}$.

gain these values referred to the LNA input are $5 \mathrm{~dB}$ and 25 $\mathrm{dB}$ above a total receiver noise of $30 \mathrm{~K}$ in $10 \mathrm{GHz}$ bandwidth.

The LNA's were connected, one at a time, to a room temperature Miteq amplifier with $19 \mathrm{~dB}$ gain driving an Agilent E4407B spectrum analyzer usually set for 2 to 20 $\mathrm{GHz}$ frequency range, 181 points with $3 \mathrm{MHz}$ resolution, but sometimes, as in Figure 9, to 1 to $6 \mathrm{GHz}$ frequency range with $1 \mathrm{MHz}$ resolution. The raw data displayed on the spectrum analyzer with absorber at $289 \mathrm{~K}$ and with cold sky, assumed to be $5 \mathrm{~K}$, is shown in Figure 9. This data is then coupled directly to a laptop computer where the noise temperature, $\mathrm{T}$ is computed by the $\mathrm{Y}$ factor method, $\mathrm{T}=$ (Thot - $\mathrm{Y}^{*}$ Tcold $) /(\mathrm{Y}-1)$ where $\mathrm{Y}$ is the ratio of hot and cold output noise powers.

The measured noise temperature results are shown in Figure 10 for data taken in Pasadena, Goldstone, and for the LNA alone. The feed plus LNA noise is $<35 \mathrm{~K}$ from 4.2 to 15 $\mathrm{GHz}$ and is $.25 \mathrm{~K}$ in most of the band. These results are consistent with the Tsys goals (on $34 \mathrm{~m}$ telescope including sky and spillover) of $<55 \mathrm{~K}$ up to $14 \mathrm{GHz}$ and $35 \mathrm{~K}$ at best frequencies. The results are good but not understood in view of the $<10 \mathrm{~K}$ LNA noise. Further tests to investigate and improve the noise temperature are planned. One question is whether the absorbing strips are at the $21 \mathrm{~K}$ temperature or are being heated by thermal radiation due to poor contact with the fins.

\section{Optics Design TradeoffS}

The antenna to be retrofitted is a 34-meter beamwaveguide antenna designed as part of the JPL Antenna Research System Task described in reference [2]. The original antenna geometry is shown in Figure 11. In contrast to a typical beamwaveguide antenna that traditionally has two curved mirrors to image a feed at the input focal point of a dual reflector antenna, for this original design, only one curved mirror - a paraboloid - is used, along with three flat mirrors. The radiation from the feed horn is allowed to spread to the paraboloid, where it is focused to a point at infinity. That is, after reflection, a collimated beam exists that is directed to the subreflector by the three flat reflectors. The energy is contained in the 2.743-m diameter of the BWG tube and does not begin to spread significantly until it exits through the main reflector. Since a collimated beam exists beyond the first mirror, this antenna is closely related to a near-field Cassegrain design, where the feed system is defined to include both the feed horn and a parabolic mirror.

In a near-field Cassegrain design, both the main reflector and the subreflector are nominally paraboloids, with dualreflector shaping used to increase the illumination efficiency on the main reflector by compensating for the amplitude taper of the feed radiation pattern. The design of the dualshaped antenna is based upon geometrical optics, with the shape of the subreflector chosen to provide for uniform amplitude illumination of the main reflector, given the distribution of the radiation striking the subreflector. The curvature of the main reflector is then modified slightly 


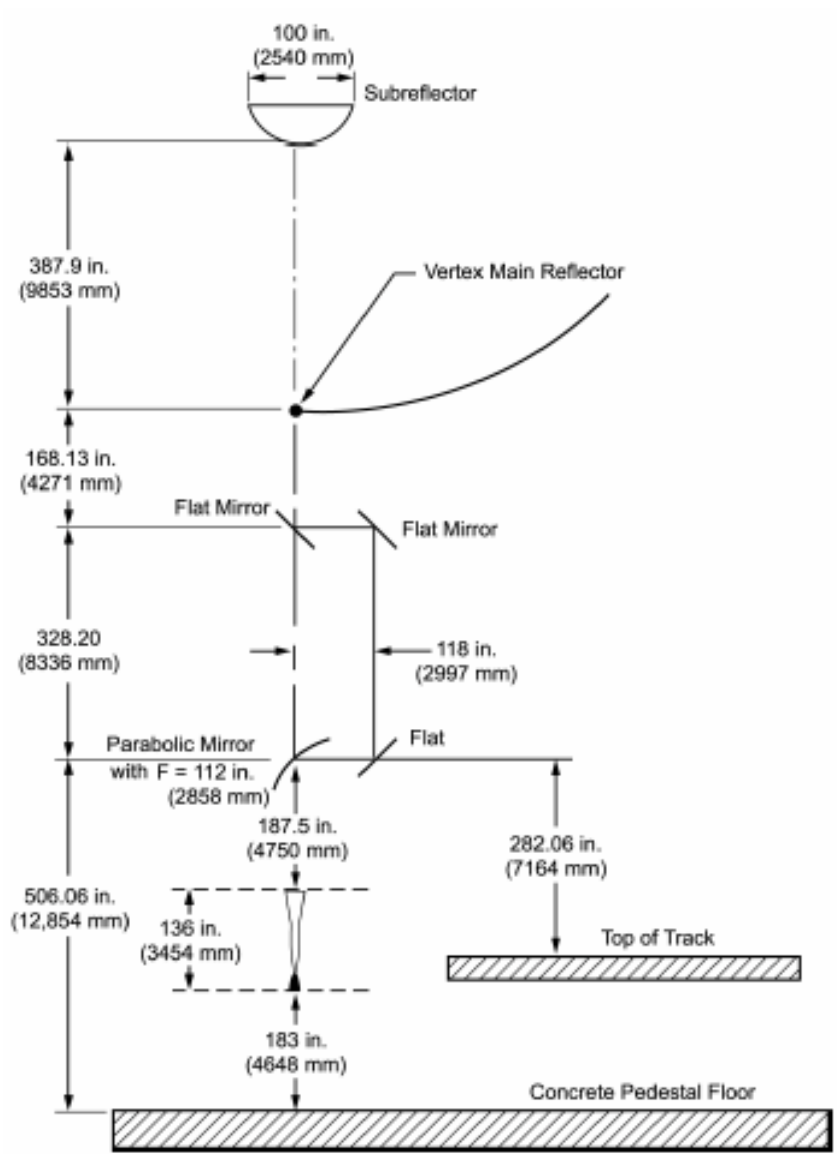

Figure 11 - Geometry of the DSS 28 Antenna

from that of the parent paraboloid to compensate for any phase errors introduced by the subreflector shaping.

The antenna was designed for high power and a narrow frequency band around 7.2 GHz. The performance at the low end of the frequency band desired for the educational program would be extremely poor if the beam waveguide system was used as part of the feed system. Hence, several redesign options to enable improved performance on the low frequency without the use of the beamwaveguide itself were examined. They included: 1) Redesigning the subreflector and using the broadband feed in a dual reflector system, 2) Using a symmetric 2.54 meter reflector tertiary reflector placed at the dish vertex 3) Using a 90 degree 2.54 meter offset design tertiary reflector at the dish vertex 4) Replacing the upper flat mirror with a parabola and placing the feed in the upper portion of the beamwaveguide tube and 5) Using a 60 degree 2.3 meter tertiary mirror at the dish vertex. Relative performance was computed using an idealized feed pattern that approximated the type of feed to be used.

Purely from an efficiency standpoint, the feed placed at the focal point of a shaped dual reflector antenna was best. However, since the optics design of the existing system did not have a useable focal point, the subreflector would have to be replaced with one with an appropriate design and the main reflector panels reset for the new subreflector design. Also, since the feed has a very broad pattern, the feed would need to be placed near the subreflector necessitating the use of a tall feed tower to support it, or hanging the feed from the subreflector. Also it would be difficult to access the feed system for maintenance and replacement. For both cost and logistical reasons, this option was rejected.

The next best performing option was a symmetric parabola placed at the vertex of the dish. Since the feed blockage would be small, the only other disadvantage of this option was that it would not be able to easily switch between feeds if, to cover the frequency band, more than one feed would be required. The offset options allowed the use of a rotating reflector that would be able to easily switch between multiple feeds. Since this is to be an educational tool, flexibility and versatility are extremely important. Hence this option was rejected.

For the offset options, the parabola at the flat mirror position performed poorly at the lowest frequencies. The performance for the other two offset options was virtually identical, so the design with the smaller parabola was selected.

\section{OPTICS DESIGN OPTIMIZATION AND Performance}

Amplitude and phase, co-polar and cross-polar patterns of the Lindgren feed were measured at fin angles of $0,45,90$, and 135 degrees and for feed rotation angles of -180 to +180 degrees in 10 degree steps. The data was taken for the feed with absorber strips on the fin outer surfaces and a metal cylinder surrounding the feed. This data was then used to optimize the design of the tertiary reflector. Frequencies of 4 and $12 \mathrm{GHz}$ were selected for optimization. The parameters to be optimized were focal length, diameter, offset height, feed tilt angle and feed defocusing. An optimization program was used and the parameters determined that yielded the highest peak gain. Using the geometry shown in Figure 12, the optimum parameters for 4 $\mathrm{GHz}$ are $\mathrm{F}=1.38 \mathrm{~m}, \mathrm{D}=2.24 \mathrm{~m}, \theta_{\mathrm{f}}=47$ degrees, $\mathrm{h}=7.7 \mathrm{~cm}$ and $\Delta \mathrm{z}=-.32 \mathrm{~cm}$. The optimum parameters for $12 \mathrm{GHz}$ are $\mathrm{F}$ $=1.27 \mathrm{~m}, \mathrm{D}=2.25 \mathrm{~m}, \theta_{\mathrm{f}}=49.3$ degrees, $\mathrm{h}=8.7 \mathrm{~cm}$ and $\Delta \mathrm{z}=$ $1.12 \mathrm{~cm}$. Data for the Lindgren feed was take every $50 \mathrm{MHz}$ from 4 to $14 \mathrm{GHz}$ and the calculated performance for the two designs shown in Figure 13. The layout in the DSS 28 reflector is pictured in Figure 14. A scaled design feed is going to be used for the 1.2 to $4 \mathrm{GHz}$ band and the parabola rotated to point to the second feed position. The performance for this lower frequency band and the scaled feed is shown in Figure 15.

\section{CONCLUSION}

The design of a wideband radio telescope to be used as part of the GAVRT educational program has been described. It 
makes use of an existing 34-meter antenna which will be retrofitted with a tertiary offset mirror placed at the apex of the main reflector. It can be rotated to use two feeds that cover the 1.2 to $14 \mathrm{GHz}$ band. The feed for 4.0 to $14.0 \mathrm{GHz}$ is a cryogenically cooled commercially available feed from
ETS-Lindgren. Coverage from 1.2 to $4.0 \mathrm{GHz}$ is provided by an un-cooled scaled version of the same feed. The performance is greater than $40 \%$ over most of the band and greater than $55 \%$ from 6 to $13.5 \mathrm{GHz}$.

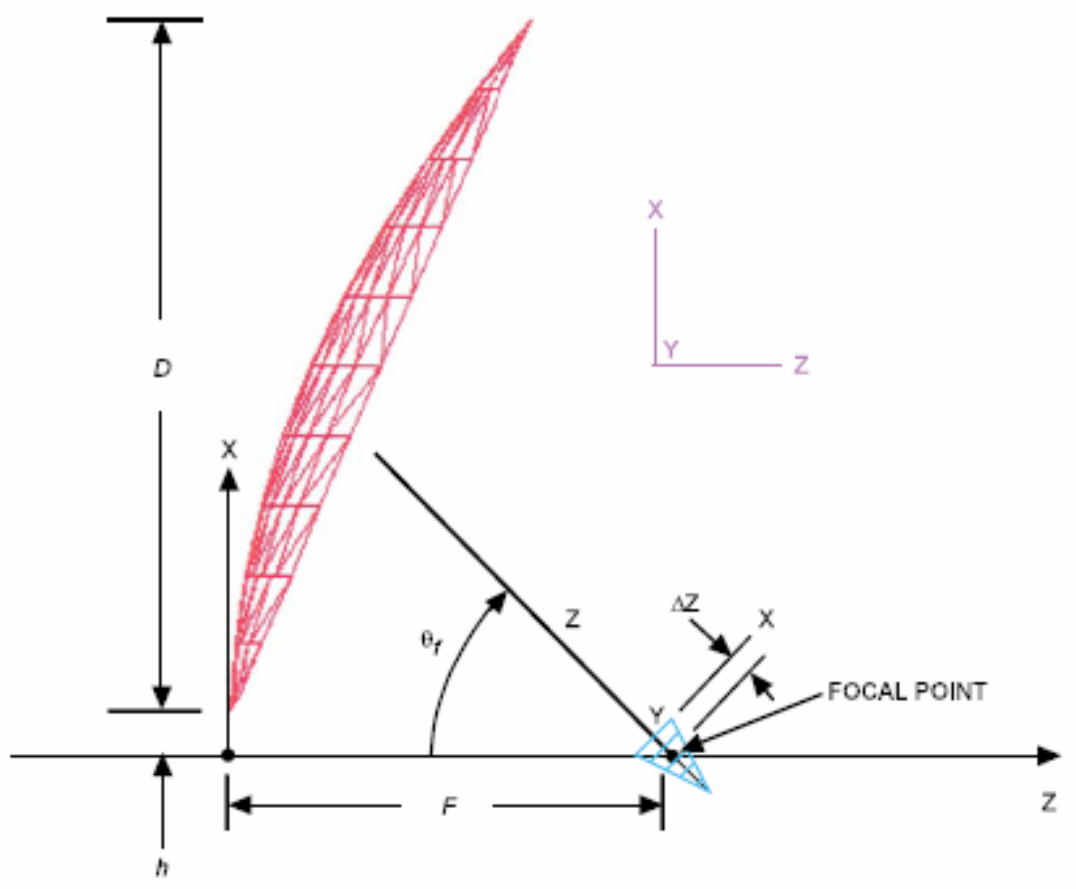

Figure 12 - Geometry of tertiary reflector

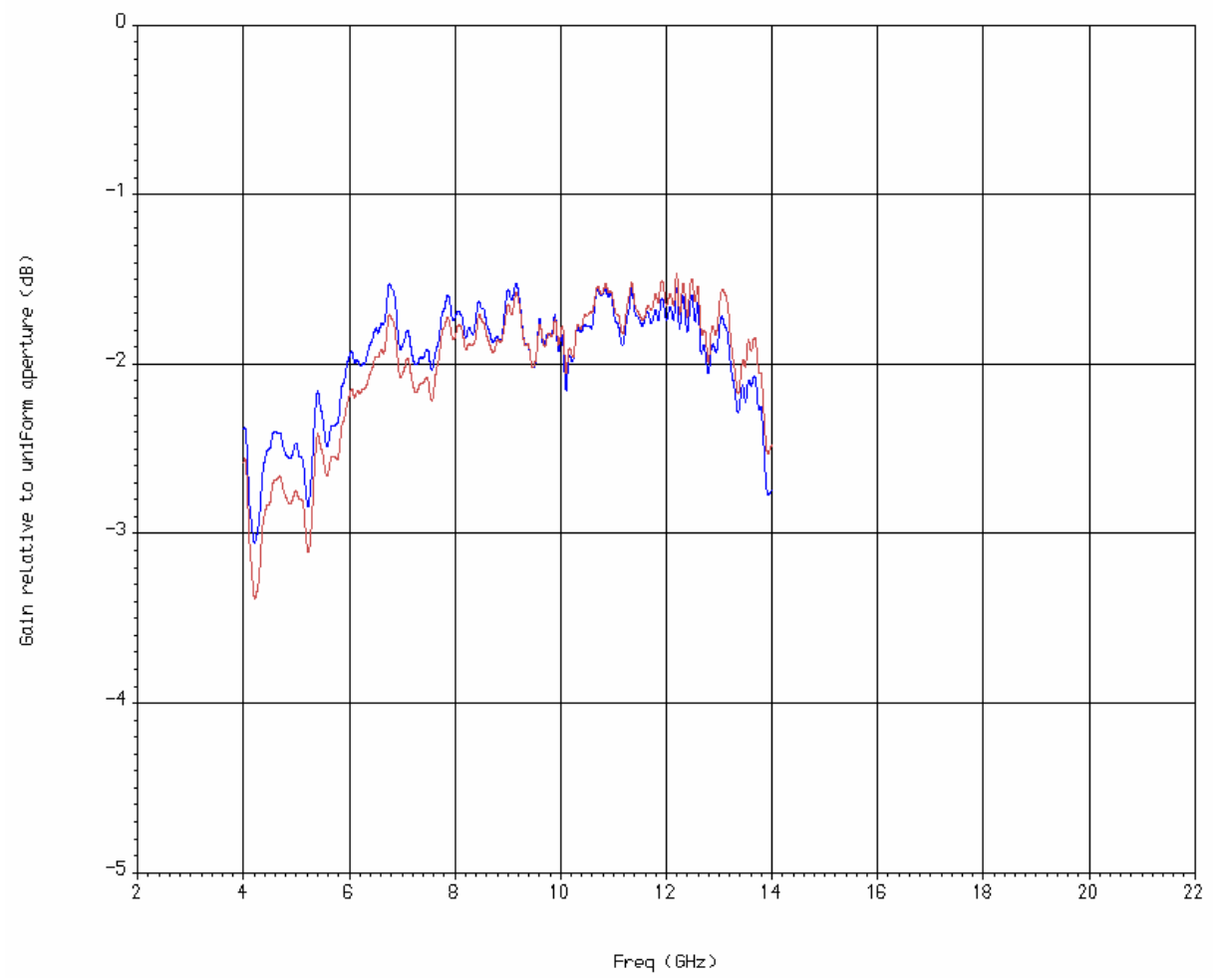

Figure 13 - Calculated peak gain performance of the cryogenically cooled Lindgren feed. Comparison of tertiary reflector optimized for $4 \mathrm{GHz}$ (blue) and $12 \mathrm{GHz}$ (red) 


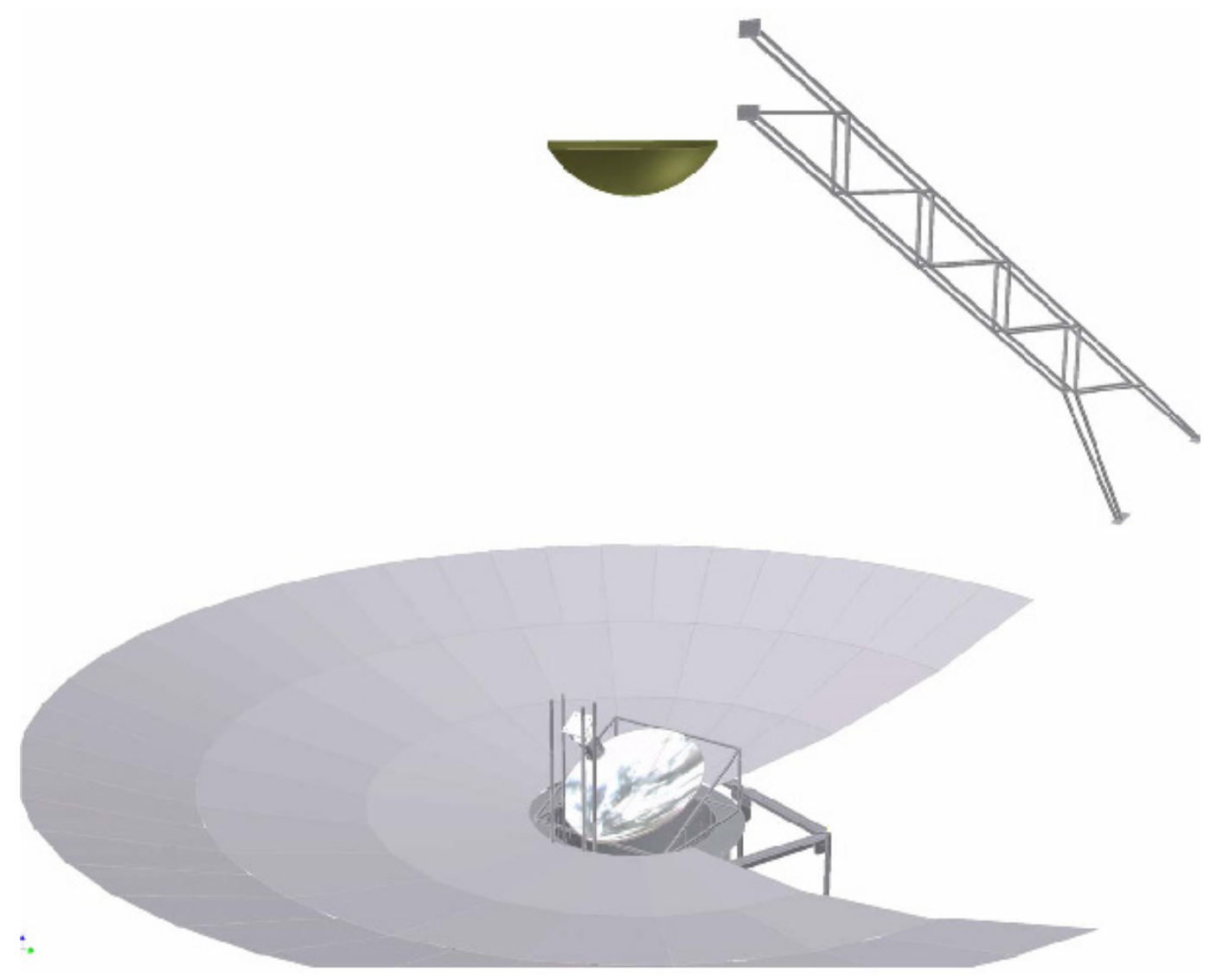

Figure 14 - Layout of tertiary mirror in main reflector

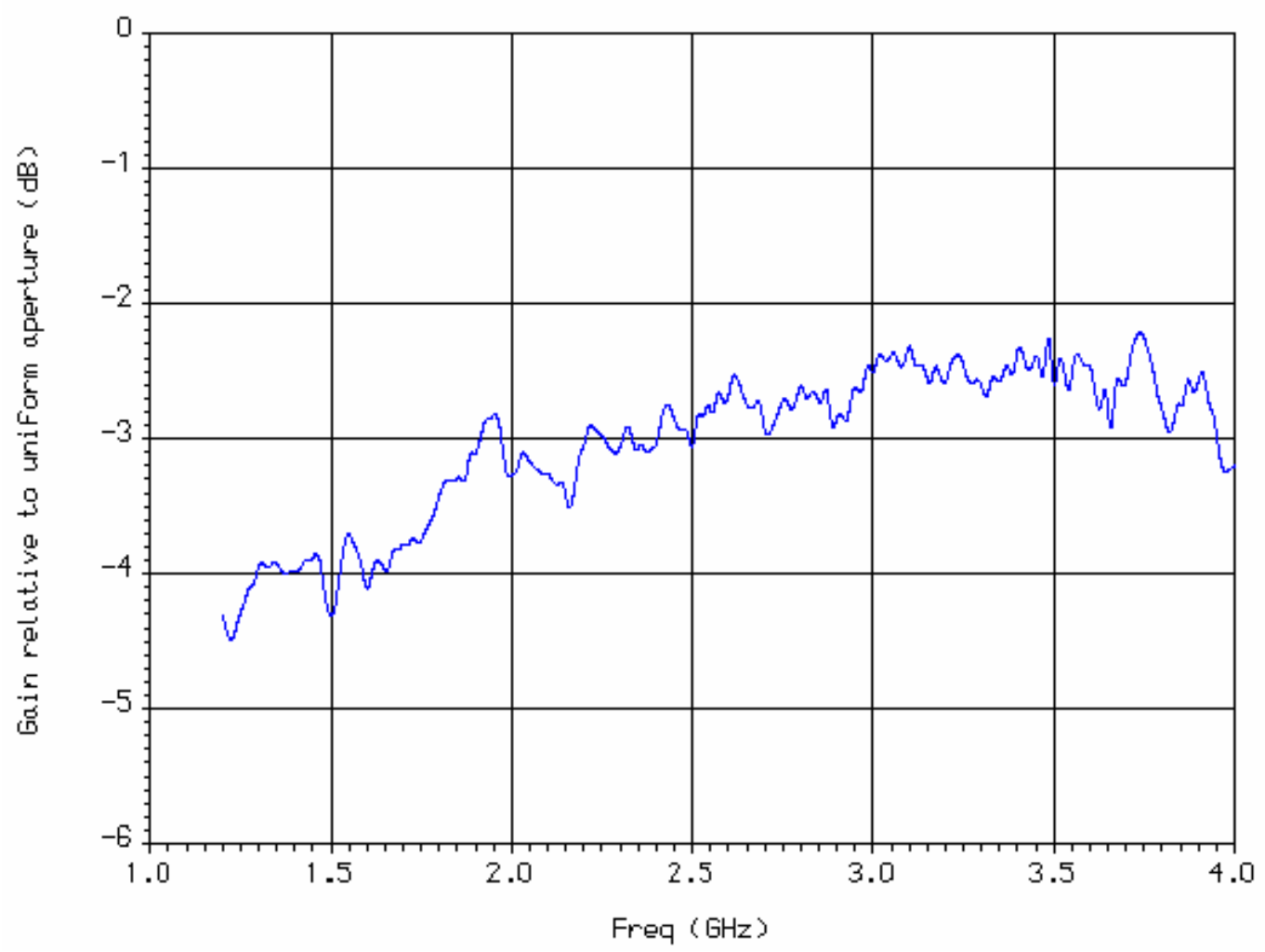

Figure 15 - Performance of the scaled un-cooled lower frequency feed 


\section{ACKNOWLEDGMENT}

The research was carried out at the Jet Propulsion Laboratory, California Institute of Technology, under a contract with the National Aeronautics and Space Administration.

\section{REFERENCES}

[1] See http://www.lewiscenter.org/gavrt/

[2] W.A. Imbriale, Large Antennas of the Deep Space Network, Wiley Interscience, 2003, Chapter 9.

[3] B.B. Burke and F. Graham-Smith, An Introduction to Radio Astronomy 2nd Edition, Cambridge Press, 2002, pp. 154-159.

[4] T. Hankins, "Nanosecond bursts from strong plasma turbulence in the Crab pulsar," Nature, vol 422, 13 March 2003.

[5] See http://kepler.nasa.gov/

[6] R. Olson, P.S. Kildal, and S. Weinreb, "The eleven antenna: a compact low-profile decade bandwidth dual polarized feed for reflector antennas." IEEE Transactions on Ant and Prop, vol 54, no 2, part 1, Feb 2006, pp. 368-375.

[7] V. Rodriguez, "A multi-octave open-boundary quadridge horn antenna for use in the $\mathrm{S}$ to Ku-bands," Microwave Journal, March, 2006, pp. 84-92.

\section{BIOGRAPHY}

William A. Imbriale is a Senior Research Scientist in the

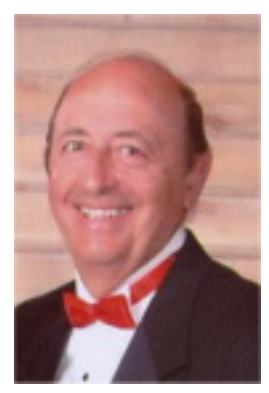

Communications Ground System Section at the Jet Propulsion Laboratory (JPL) in Pasadena, California. Since starting at JPL in 1980, he has led many advanced technology developments for large ground-station antennas, lightweight spacecraft antennas, and millimeterwave spacecraft instruments. He is currently working on a technology contract for the Earth Sciences Technology Office (ESTO) to develop a subreflector consisting of MEMS switches integrated with patch reflect array elements that will compensate, in real time, for on-orbit distortions of a membrane inflatable antenna. He is also the lead engineer for the Spanish supplied High Gain Antenna System for the Mars Science Laboratory (MSL) rover. Earlier positions at JPL have included being the Assistant Manager for Microwaves in the Ground Antennas and Facilities Engineering Section and the Manager of the Radio Frequency and Microwave Subsystem Section.
Prior to joining JPL in 1980, Dr. Imbriale was employed at the TRW Defense and Space Systems Group where he was the Subproject Manager for the Antennas of the TDRSS program.

Dr. Imbriale is a Fellow of the IEEE and has an extensive list of publications.

Sander Weinreb is presently a Principal Scientist at JPL

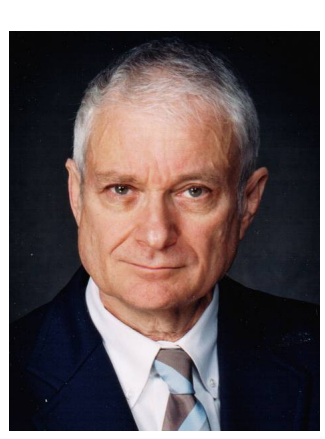
and a Faculty Associate at Caltech. Prior to this he was a Research Professor in the Department of Physics and Astronomy at $U$. of Massachusetts. His main present area of research is the development of low-noise microwave and millimeter wave integrated circuits (MMICs) for use in radio astronomy and atmospheric research. Prior to joining $U$. of Mass., during 1989-1996, he was Principal Scientist and Leader of the Millimeter-Wave Design and Test Group at Martin Marietta Laboratories where he led the design of millimeter-wave MMICs and prototype radar and radiometer systems. During 1988 and 1989 he was a visiting professor at $U$. of Virginia. Prior to this he was Head of the Electronics Division (1965-1985) and Assistant Director (1985-1988) at National Radio Astronomy Observatory where he was responsible for the design, construction, operation, and maintenance of radio astronomy receivers at the Green Bank, WV, and Kitt Peak, $A Z$ observatories. At NRAO he led the group responsible for the design of the electronics system for the Very Large Array. Dr. Weinreb received the B.S.E.E. and Ph.D. degrees from M.I.T. in 1958 and 1963, respectively. He is the author of over 120 publications in the areas of digital correlation techniques, radio astronomy observations, array receivers, and low-noise amplifiers.

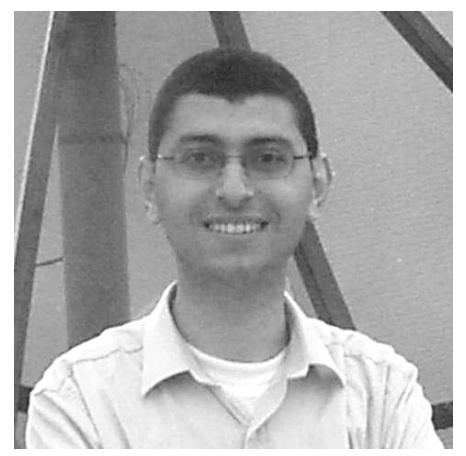

Hamdi Mani was born in Hammam Sousse, Tunisia on September 18, 1980. After graduating from secondary school with high marks he has selfeducated himself in the area of radio astronomy and microwave receivers. In 2005 he was hired as a Research Technician in the Caltech Electrical Engineering Department where he is presently employed. He specializes in the construction and testing of MMIC cryogenically-cooled very low noise amplifiers, microwave measurements, and the integration of radio astronomy receivers. 